\title{
Artigo/Article
}

\section{Estudo clínico-radiológico do esôfago e dos cólons na fase aguda da doença de Chagas com relato de três casos de remissão espontânea de aperistalse do esôfago do grupo I}

\author{
Clinical and radiological evaluation of the esophagus and colon in the acute phase of Chagas \\ disease with Report of three cases of spontaneous remission of esophageal group I aperistalsis
}

\section{Anis Rassi ${ }^{1}$ e Joffre Marcondes de Rezende ${ }^{1,2}$}

\section{RESUMO}

Introdução: $\mathrm{O}$ esôfago e os cólons foram investigados em pacientes na fase aguda da doença de Chagas, entre 1956 e 1989. Métodos: A deglutição e o exame radiológico do esôfago foram explorados em 94 (90,4\%) pacientes, sendo excluídas 10 crianças em virtude de tenra idade. O enema opaco foi realizado em 59 (56,7\%) pacientes. Resultados: A deglutição foi referida como normal em $86(91,5 \%)$ pacientes, 5 dos quais apresentaram aperistalse do grupo I, enquanto a disfagia incipiente foi referida por 8 , dos quais 7 apresentaram exame radiológico normal e apenas um, aperistalse do grupo I. Um segundo exame radiológico, realizado em 4 dos 6 casos, 6 meses após o primeiro, mostrou-se normal em 3 e permaneceu inalterado em um, o único que recebeu tratamento (benzonidazol) e considerado como curado da infecção. O ritmo intestinal resultou normal em 96 (92,3\%) pacientes, obstipado em 7 (6,7\%) e diarreico em um (1\%). O enema evidenciou resultado normal em 54 (91,5\%) pacientes, dolicossigmoide em $4(6,8 \%)$ e dolicorretomegassigmoide em um $(1,7 \%)$, que a eletromanometria demonstrou ser de natureza funcional. Conclusões: Para explicar a regressão da aperistalse, aventaram os autores duas hipóteses que não se excluem. A primeira,é a de que o processo inflamatório intramural na fase inicial da infecção poderia interferir na motilidade esofagiana, enfraquecendo a onda peristáltica; com a regressão do processo inflamatório, o peristaltismo voltaria ao normal, desde que a desnervação fosse limitada; a segunda hipótese é a de que tenha havido real re-inervação, com recuperação normal da onda peristáltica.

Palavras-chaves: Doença de Chagas. Fase aguda. Esôfago e cólons.

\begin{abstract}
Introduction: The esophagus and colons of patients with acute Chagas disease were studied. Methods: Deglutition and radiological examination of the esophagus were assessed in 94 (90.4\%) patients, 10 children were excluded due to their age. Intestinal transit was assessed in 59 (56.7\%) patients. Results: Deglutition was normal in 86 (91.5\%) patients, 5 of whom presented aperistalsis (group I). Incipient dysphagia was reported by 8 patients, 7 of whom normal barium swallowing, while only one presented esophageal aperistalsis (group I). A second radiological examination of the esophagus was performed in 4 of the 6 cases with aperistalsis 6 months after the initial test. It was normal in 3 , and showed aperistalsis in one case, the only patient who was treated with benznidazole and considered cured of the infection. Intestinal transit was normal in $96(92.3 \%)$ patients, constipated in 7 (6.7\%), and diarrheic in one (1\%). Barium enema was normal in 54/59 (91.5\%), showed a dolichosigmoid in $4(6.8 \%)$ and a dolichorectomegasigmoid in one (1.7\%), of functional origin, according to the electromanometric results. Conclusions: To explain the regression of aperistalsis, 2 non-excluding hypothesis were postulated: the intramural inflammatory process, established during the acute phase, could interfere with esophageal motility by weakening the peristaltic waves; with the remission of the inflammatory reaction, the peristaltic activity of the esophagus would return to normal, as long as no extensive denervation occurred; and/or a definitive reinnervation is responsible for the recuperation of the normal esophageal peristaltic activity.
\end{abstract}

Keywords: Chagas disease. Acute phase. Esophagus and colon.

1. Professor Emérito, Faculdade de Medicina, Universidade Federal de Goiás, Goiânia, GO. 2. Professor Honoris Causa, Universidade de Brasília, Brasília, DF.

Endereço para correspondência: Dr. Anis Rassi. Hospital Anis Rassi. Av. José Alves 453, Setor Oeste, 74110-020 Goiânia, GO.

Tel: 5562 3227-9000, Fax: 5562 3227-9121

e-mail: anisrassi@arh.com.br

Recebido para publicação em 31/03/2010

Aceito em 13/10/2010

\section{INTRODUÇÃO}

O comprometimento da inervação intrínseca do trato digestivo na doença de Chagas acha-se definitivamente comprovado e aceito como causa das alterações motoras que conduzem à formação do megaesôfago e do megacólon. Por sua localização intramural, o plexo mioentérico de Auerbach é mais atingido do que o de Meissner.

Koeberle demonstrou que a desnervação ocorre precocemente, já na fase aguda da infecção; admitiu também que a desnervação da fase aguda fosse mais importante do que a da fase crônica na determinação da evolução da enfermidade; expressou este ponto de vista em uma frase que se tornou bem conhecida, a de que $o$ destino do chagásico decide-se na fase aguda ${ }^{1}$.

Outros pesquisadores comprovaram igualmente o acometimento dos plexos intramurais na fase aguda da doença ${ }^{2-4}$.

Embora em estudos anatomopatológicos seja evidente a desnervação na fase aguda da doença de Chagas, são raras as manifestações clínicas, neste período, decorrentes da mesma. De modo geral, o aparecimento do megaesôfago e do megacólon (segmentos do tubo digestivo mais comumente atingidos na doença de Chagas) dá-se alguns anos depois da infecção, o que poderia ser explicado pelo prosseguimento lento da desnervação durante a fase crônica, aliado à diminuição do número de neurônios que ocorre naturalmente com o envelhecimento ${ }^{5}$.

Por outrolado, o acompanhamento dos pacientes, a longo prazo, evidencia que apenas parte deles, cerca de $10 \%$, desenvolve megaesôfago e/ou megacólon como manifestação da doença ${ }^{6}$.

Segundo Koeberle ${ }^{5}$, para que ocorram o megaesôfago e o megacólon, é necessária uma desnervação da ordem de $90 \%$ para o esôfago e de $55 \%$ para o cólon. Adad e cols ${ }^{7}$, em estudos quantitativos dos neurônios do cólon sigmoide, em 17 casos de megacólon chagásico, encontraram uma desnervação muito próxima do valor estabelecido por Koeberle, 
estando acima de 55\%, em 76,5\% dos casos; verificaram também que a desnervação é mais intensa na junção retossigmoidiana.

São escassos na literatura estudos de pacientes na fase aguda com vistas à detecção de alterações motoras do esôfago e do cólon ${ }^{8}$.

Chagas", em seu trabalho sobre fase aguda, assinalou: "Outro sinal que nos foi revelado por infecção aguda em adulto, é o que se expressa em acentuada disfajia, para os alimentos solidos e tambem para os liquidos. A injestão de substancias solidas exije, para se completar, o auxilio de agua, referindo os doentes que o alimento fica retido no esofago, trazendo sensações penosissimas. Mesmo a injestão de substancias liquidas, e, da propria agua, pode apresentar dificuldade, não raro invenciveis, necessitando $o$ artificio de deglutições cuidadosas e de pequenas parcelas do liquido.

Este sinal, de patojenia ainda mal esclarecida, relaciona-se talvez com a condição de disfajia conhecida pelo nome de mal de engasgo, endemia extensa em rejiões do interior do Brazil e, segundo nossas observações, verificada principalmente naquelas zonas, onde é encontrada a tripanosomiase. Será o mal de engasgo um elemento mais da tripanosomiase brazileira e essa disfajia das fórmas agudas traduzirá a fase inicial da sindrome? Observámos, no correr de nossos estudos em Lassance, numero bem elevado de doentes com mal de engasgo, havendo, em todos eles, outros sinais simultaneos da tripanosomiase. Apesar disso, tornam-se precisas novas pesquizas que autorizem, de modo inrrecusavel, incluir o mal de engasgo na sintomatolojia multiforme da infecção pelo Trypanosoma Cruzi".

Versiani e Junqueira ${ }^{10}$ relataram disfagia em um caso de fase aguda de evolução prolongada, febril durante meses e com formas amastigotas do T. cruzi em linfonodo. Koeberle ${ }^{11}$ teve a oportunidade de necropsiar dois pacientes falecidos durante a fase aguda, encontrando $70 \%$ de desnervação do esôfago num caso e $90 \%$ no outro; entretanto, não fez referência à disfagia. Num terceiro caso, de criança que adquiriu a infecção aos 9 meses, "no fim da fase aguda a deglutição foi extremamente difícil e a defecação foi possível somente após um enema. Logo se desenvolveu um megaesôfago e um megacolon. A criança morreu no pós-operatório da operação de Heller". Becker ${ }^{12}$ descreveu um caso agudo letal, em uma paciente de 40 anos, sob uso de corticoide e que adquiriu a infecção por transfusão de sangue, no qual apareceu disfagia. $\mathrm{O}$ exame anatomopatológico revelou uma desnervação da ordem de $90 \%$ no esôfago. Dias ${ }^{13}$, à anamnese dirigida, assinalou a ocorrência de disfagia em $4(5,6 \%)$ dentre 71 pacientes na fase aguda, em dois deles, acompanhada de regurgitação. $\mathrm{O}$ exame radiológico, realizado em um dos casos, decorridos um ano e 10 meses de doença, revelou "megaesôfago grau I-II."

\section{MÉTODOS}

Compõem a casuística 104 pacientes que foram atendidos na fase aguda da doença de Chagas, de transmissão vetorial, parasitologicamente comprovada, entre 1956 e 1989.

A deglutição foi apurada pelo interrogatório sintomatológico, com as clássicas perguntas, entre nós: "engole bem a comida" e "comida fria entala na boca do estômago?" Se a resposta era afirmativa para disfagia, indagávamos sobre os demais sintomas que a acompanham. O ritmo intestinal foi considerado normal quando as evacuações se processavam diariamente ou de dois em dois dias e obstipado quando 3 ou mais dias separavam uma da outra.
O exame radiológico do esôfago foi sempre realizado segundo a técnica de Lauar e cols ${ }^{14}$, segundo a qual são tomadas duas radiografias: a primeira, imediatamente após a ingestão do meio de contraste e a segunda, um minuto depois da primeira. Considerouse normal o esôfago que não apresentasse retenção do contraste na segunda radiografia. O estudo radiológico do cólon por enema opaco com duplo contraste obedeceu à técnica clássica.

A distribuição dos enfermos por sexo, idade, mês de ocorrência da infecção, manifestações clínicas, resultados do eletrocardiograma e do exame radiológico do coração, esôfago e cólons, acham-se consignados em trabalho anterior ${ }^{15}$. Acresce informar que os pacientes eram procedentes do Estado de Goiás e zonas limítrofes (Estados do Tocantins, Minas Gerais e Bahia).

No trabalho citado, demos conta do resultado do exame radiológico do esôfago e dos cólons sem fazer referência à sintomatologia, informando que pormenores do estudo seriam motivos de futura publicação, que agora apresentamos.

\section{RESULTADOS}

A deglutição e o exame radiológico do esôfago foram investigados em 94 (90,4\%) dos 104 pacientes, deixando de ser feito em 10, por tratar-se de crianças de tenra idade. A deglutição foi referida como normal por 86 (91,5\%), 5 dos quais apresentaram aperistalse do esôfago do grupo I, enquanto a disfagia para alimentos sólidos, quando frios (principalmente se ingeridos rapidamente e, geralmente, o arroz), foi informada por 8 , dos quais 7 apresentaram exame radiológico normal e apenas um, aperistalse do grupo I (caso 4).

O ritmo intestinal foi apurado em todos os 104 pacientes (nas crianças de tenra idade, por informação da mãe). Resultou normal em 96 (92,3\%), obstipado em 7 (6,7\%), dois dos quais desde antes da fase aguda, e diarreico em um (1\%). O enema opaco com duplo contraste pôde ser realizado em 59 (56,7\%) dos 104 pacientes. Mostrou resultado normal em 54 (91,5\%), dolicossigmoide em $4(6,8 \%)$, todos com ritmo intestinal normal e dolicorretomegassigmoide em um (1,7\%), também com ritmo intestinal normal e que a eletromanometria revelou tratar-se de alteração funcional.

A Tabela 1 mostra que dos 6 pacientes que apresentaram aperistalse do esôfago do grupo I, 3 (casos 1, 3 e 4) eram do sexo masculino e 3 (casos 2,5 e 6 ) do feminino. É de assinalar-se que em 5 casos, os de números 2, 3, 4, 5 e 6, a aperistalse apareceu em plena fase aguda, coincidente com quadro clínico próprio da mesma e com exame direto do sangue positivo para T. cruzi. Já no caso 1 , a aperistalse se fez presente no $5^{\circ}$ mês de doença, em consonância com remissão da sintomatologia da fase aguda. Um segundo exame radiológico, realizado em 4 (casos 1, 2, 3 e 5) desses 6 pacientes, cerca de 5 meses após, mostrou-se normal em 3 (Figuras 1, 2 e 3 ) e permaneceu inalterado em uma (caso 5) paciente, a única dos 4 que recebeu tratamento específico (benzonidazol) e considerada curada da infecção pela negativação, repetidas vezes, das provas sorodiagnósticas e do xenodiagnóstico. 
TABELA 1 - Dados dos pacientes que apresentaram aperistalse do esôfago do grupo I.

\begin{tabular}{|c|c|c|c|c|c|c|}
\hline Caso & Identificação & $\begin{array}{c}\text { Tempo de } \\
\text { doença }\end{array}$ & Quadro clínico & $\begin{array}{c}\text { Trypanosoma } \\
\text { cruzi }^{*}\end{array}$ & Disfagia & Rx do esôfago \\
\hline \multirow[t]{4}{*}{1} & M 264.56 & 30 dias & febre chagoma & positiva & não & normal \\
\hline & Masculino & $5^{\circ}$ mês & $\mathrm{Ndr}$ & $\ldots$ & não & aperistalse \\
\hline & 32 anos & $12^{\circ}$ mês & $\mathrm{Ndr}$ & $\cdots$ & não & normal \\
\hline & Lavrador & & & & & \\
\hline \multirow[t]{4}{*}{2} & M 265.56 & 22 dias & febre romaña & positiva & não & aperistalse \\
\hline & Feminino & & edema & & & \\
\hline & 21 anos & $5^{\circ}$ mês & $\mathrm{Ndr}$ & $\ldots$ & não & normal \\
\hline & Doméstica & & & & & \\
\hline \multirow[t]{4}{*}{3} & M 256.60** & 19 dias & febre chagoma & positiva & não & aperistalse \\
\hline & Masculino & & hipertrofia de & & & \\
\hline & 52 anos & & linfonodos & & & \\
\hline & Lavrador & $6^{\circ}$ mês & $\mathrm{Ndr}$ & $\ldots$ & não & normal \\
\hline \multirow[t]{4}{*}{4} & M $3.57^{* * *}$ & 17 dias & febre chagoma & positiva & $\operatorname{sim}$ & aperistalse \\
\hline & Masculino & & edema & & & \\
\hline & 52 anos & & & & & \\
\hline & Lavrador & & & & & \\
\hline \multirow[t]{4}{*}{5} & M 441.74 & 32 dias & febre & positiva & não & aperistalse \\
\hline & Feminino & & hepato-esplenomegalia & & & \\
\hline & 41 anos & & edema & & & \\
\hline & Doméstica & até o $17^{\circ}$ ano & $\mathrm{Ndr}$ & $\ldots$ & $\operatorname{sim}$ & aperistalse \\
\hline \multirow[t]{4}{*}{6} & M 435.80 & 30 dias & febre chagoma & positiva & não & aperistalse \\
\hline & Feminino & & hipertrofia de linfonodos & & & \\
\hline & 61 anos & & edema & & & \\
\hline & Doméstica & & & & & \\
\hline
\end{tabular}

Ndr: Nada digno de registro* ${ }^{*}$ esquisa do Trypanosoma cruzi no sangue entre lâmina e lamínula. ${ }^{* *}$ Submetido à tentativa de tratamento específico pelo Prof. José Ferreira Fernandes (HC da FM da USP) com aminonucleosídeo da estilomícina associado à primaquina. Como efeito colateral apresentou síndrome nefrótica (reversível); não se curou da infecção chagásica. ${ }^{* * *}$ Suicídio em 1964.

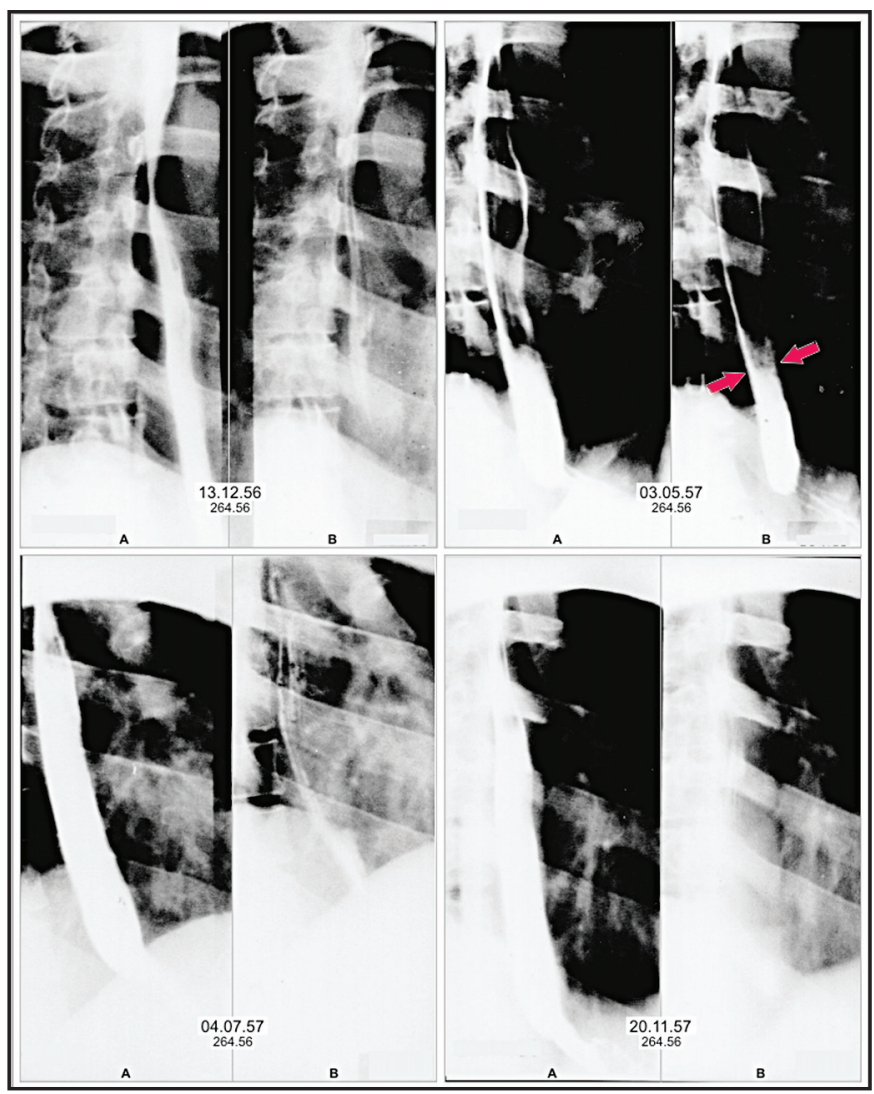

FIGURA 1 - Montagem fotográfica de radiografias do esôfago do caso 1 em quatro ocasiões. Paciente Matr. 264.56.

13.12.56: prova de retenção negativa (esôfago radiologicamente normal), 3.5.57: aspecto típico de aperistalse do grupo I (as setas apontam para o nível de retenção do meio de contraste), 4.7.57: retenção mínima do meio de contraste, 20.11.57: esvaziamento completo do esôfago na prova de retenção (esôfago radiologicamente normal), A: radiografia obtida imediatamente após a deglutição do meio de contraste, B: radiografia tomada um minuto após.

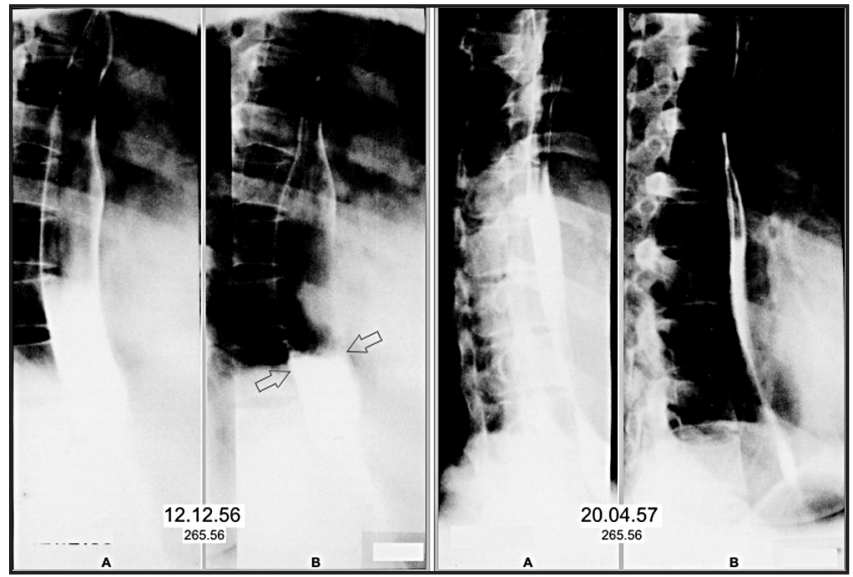

FIGURA 2 - Montagem fotográfica de radiografias do esôfago do caso 2 em duas ocasióes. Paciente Matr. 265.56.

12.12.56: aspecto típico de aperistalse do esôfago do grupo I, 20.4.57: observase mínima quantidade residual do meio de contraste, 'compatível com exame radiológico normal, A: radiografia obtida imediatamente após a deglutição do meio de contraste, B: radiografia tomada um minuto após. 

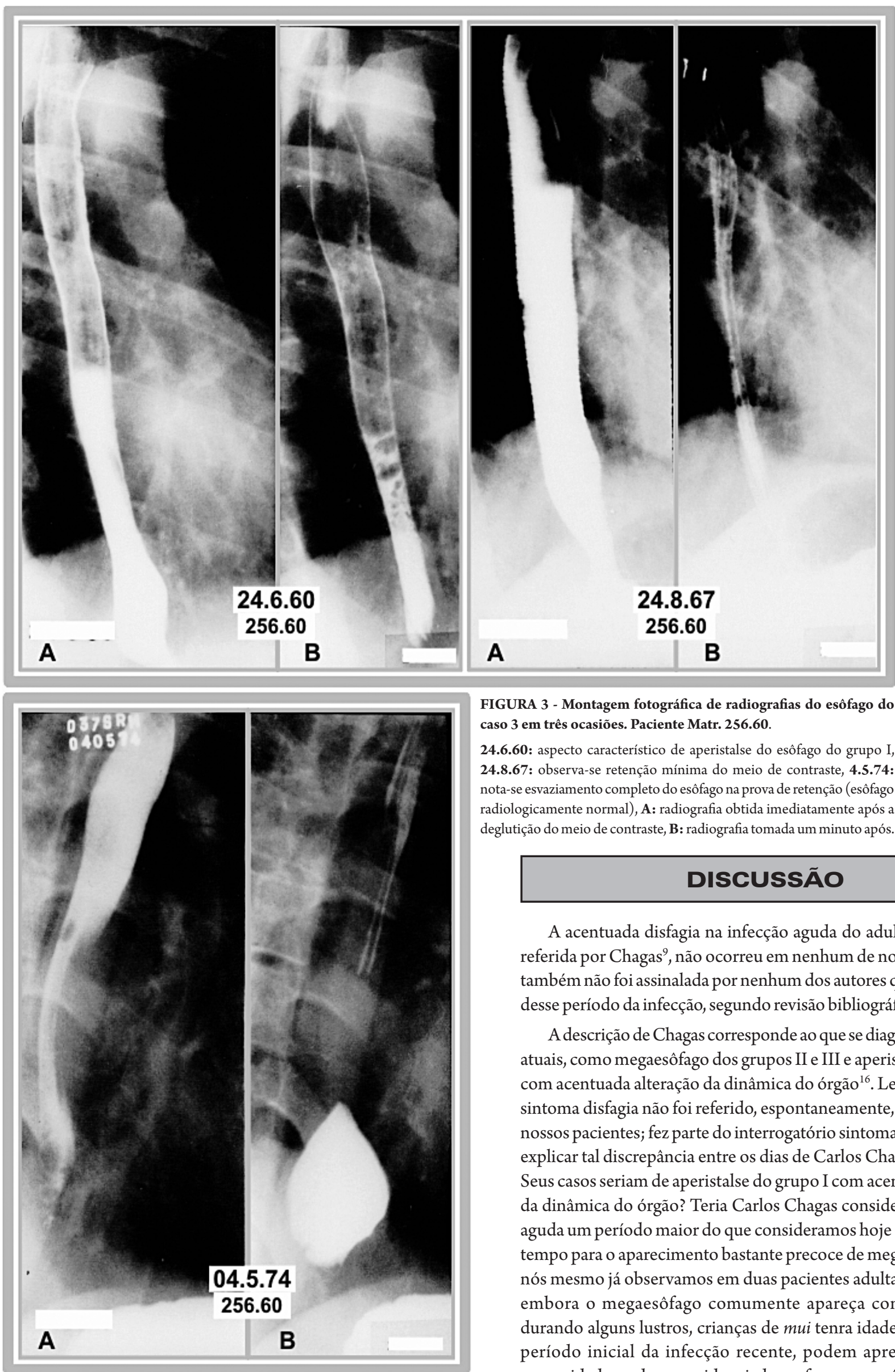

FIGURA 3 - Montagem fotográfica de radiografias do esôfago do caso 3 em três ocasiões. Paciente Matr. 256.60.

24.6.60: aspecto característico de aperistalse do esôfago do grupo I, 24.8.67: observa-se retenção mínima do meio de contraste, 4.5.74: nota-se esvaziamento completo do esôfago na prova de retenção (esôfago radiologicamente normal), A: radiografia obtida imediatamente após a deglutição do meio de contraste, B: radiografia tomada um minuto após.

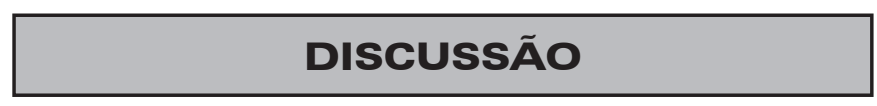

A acentuada disfagia na infecção aguda do adulto, tal como foi referida por Chagas $^{9}$, não ocorreu em nenhum de nossos pacientes e também não foi assinalada por nenhum dos autores que se ocuparam desse período da infecção, segundo revisão bibliográfica que fizemos.

A descrição de Chagas corresponde ao que se diagnostica, nos dias atuais, como megaesôfago dos grupos II e III e aperistalse do grupo I com acentuada alteração da dinâmica do órgão ${ }^{16}$. Lembramos que 0 sintoma disfagia não foi referido, espontaneamente, por nenhum de nossos pacientes; fez parte do interrogatório sintomatológico. Como explicar tal discrepância entre os dias de Carlos Chagas e os nossos? Seus casos seriam de aperistalse do grupo I com acentuada alteração da dinâmica do órgão? Teria Carlos Chagas considerado como fase aguda um período maior do que consideramos hoje e, assim, haveria tempo para o aparecimento bastante precoce de megaesôfago, como nós mesmo já observamos em duas pacientes adultas ${ }^{17,18}$ ? Também, embora o megaesôfago comumente apareça com a infecção já durando alguns lustros, crianças de mui tenra idade, portanto, com período inicial da infecção recente, podem apresentá-lo e essa precocidade pode ser evidenciada na forma congênita da doença de Chagas. Necrópsias realizadas em casos de transmissão vertical 
demonstraram lesões no trato digestivo caracterizadas por infiltrado inflamatório e degeneração de neurônios do plexo mioentérico ${ }^{19}$. Nos casos de sobrevida do recém-nato, foram registrados sintomas de aperistalse do esôfago, como disfagia, regurgitações e eventuais episódios de aspiração traqueobrônquica. Atias e Almonte ${ }^{20}$ relataram um caso em que os sintomas apareceram aos 11 meses de idade; a radiografia evidenciou megaesôfago do grupo III e o paciente foi tratado com dilatações por sonda de Hurst e mantido em dieta semisólida. Tafuri e cols ${ }^{21}$ descreveram caso de sobrevida de 6 meses e 21 dias, que apresentava regurgitações, interpretadas como vômitos, e desnutrição. O exame radiológico mostrou megaesôfago "do grau II ou III", com prova farmacológica de desnervação pelo teste da metacolina positivo. Apesar disso, as lesões do plexo mioentérico foram consideradas discretas pelos autores. Bittencourt ${ }^{22}$, dentre 33 casos de transmissão vertical, encontrou 3 recém-nascidos que sobreviveram até a idade de 2, 5 e 6 meses, os quais apresentaram "disfagia" e regurgitações, sendo que um dos lactentes, com megaesôfago do grupo III chegou a ser submetido à operação de Heller.. Na casuística de megaesôfago de um dos autores (AR), há um caso com 20 meses de idade, com xenodiagnósticos e provas sorológicas para doença de Chagas positivos, sem antecedente de transfusão de sangue, filha de mãe com provas sorológicas negativas para doença de Chagas, apresentando megaesôfago do gupo III, com sintomatologia iniciada aos 16 meses de vida (trabalho em publicação).

Há que assinalar, ainda, o fato paradoxal da deglutição ter sido referida como normal nos 5 pacientes com aperistalse do grupo I, enquanto disfagia incipiente foi informada por 7 entre 8 enfermos com exame radiológico normal; nesses últimos, a eletromanometria do órgão com a prova farmacológica de desnervação (método mais sensível que a radiologia para detecção de alteração motora) talvez evidenciasse algum grau de comprometimento da dinâmica esofágica.

Com relação ao cólon, a detecção de alterações radiológicas ou de obstipação atribuíveis à infecção chagásica na fase aguda é dificultada pela própria anatomia e fisiologia desse segmento. Do ponto de vista radiológico, há uma grande variação anatômica quanto ao comprimento e ao diâmetro do cólon, que não permite distinguir entre o normal e o patológico nos casos incipientes. Além disso, a grande distensibilidade de suas paredes propicia a dilatação secundária à estase fecal de qualquer natureza, como a que se observa no chamado megacólon funcional ou psicogênico da infância. Por outro lado, a obstipação é um sintoma inespecífico não confiável para avaliação do comprometimento precoce do cólon na infecção chagásica. Assim sendo, o achado de dolicossigmoide em 4 casos e de dolicorretomegassigmoide em um caso não significa, necessariamente, comprometimento do cólon pela doença de Chagas. Cumpre assinalar que no caso de dolicorretomegassigmoide a eletromanometria revelou tratar-se de alteração funcional. Quanto à obstipação, dos 7 pacientes que referiram este sintoma, dois já o apresentavam antes da fase aguda. O método mais indicado de investigação nessa fase seria a eletromanometria com a prova farmacológica de desnervação, tal como foi realizado pelo Professor Helio Moreira em 6 dos casos dessa casuística, um deles com resultado positivo e que apresentava ritmo intestinal e enema opaco normais, conforme referimos em trabalho anterior ${ }^{8}$.

A regressão da aperistalse do grupo I verificada em 3 pacientes ao exame radiológico, imediatamente após a fase aguda, poderia fazer pensar, em primeiro lugar, em falha técnica. Contudo, o mesmo fato foi relatado em estudos longitudinais por $\operatorname{Pompeu}^{23} \mathrm{em}$ dois casos, e por Castro e cols ${ }^{24} \mathrm{em} 4$ casos. Duas outras hipóteses, que não se excluem, podem ser levantadas; a primeira é a de que o processo inflamatório intramural na fase inicial da infecção poderia interferir na motilidade esofagiana, enfraquecendo a onda peristáltica e ocasionando a retenção residual do meio de contraste no segmento distal do esôfago; com a regressão do processo inflamatório agudo, o peristaltismo voltaria ao normal, desde que a desnervação fosse limitada, de modo a não comprometer a motilidade do órgão, como ocorre, aliás, em cerca de $90 \%$ dos chagásicos. A segunda hipótese é de que haja realmente re-inervação, com recuperação da amplitude normal da onda peristáltica. Conforme foi comprovado experimentalmente em trabalhos recentes, o plexo mioentérico tem capacidade de regeneração em áreas desnervadas pelo cloreto de benzalcônio, que produz desnervação semelhante a que ocorre na doença de Chagas. A re-inervação tem início a partir dos neurônios preservados de áreas vizinhas, os quais emitem fibras pós-ganglionares ao tecido muscular da área desnervada. Segundo Hanani e cols ${ }^{25}$, pode haver ainda a formação de novos neurônios, contrariamente ao que se acreditava.

É óbvio que, se houver destruição extensa do plexo, a re-inervação é inviável, porém é perfeitamente admissível que ela ocorra quando a desnervação é limitada e há neurônios íntegros nas imediações das áreas atingidas.

\section{CONFLITO DE INTERESSE}

Os autores declaram não haver nenhum tipo de conflito de interesse no desenvolvimento do estudo.

\section{REFERÊNCIAS}

1. Koeberle F. Patogenia da moléstia de Chagas. Estudo dos órgãos musculares ôcos. Rev Goiana Med 1957; 3:155-180.

2. Tafuri WL, Brener Z. Lesões do sistema nervoso autônomo do camundongo albino na tripanosomíase experimental na fase aguda. O Hospital 1966; 69:371-383.

3. Andrade SG, Andrade ZA. Doença de Chagas e alterações neuronais no plexo de Auerbach (estudo experimental em camundongos). Rev Inst Med trop São Paulo 1966; 8:219-224.

4. Paiva SM. Desnervação do esôfago na fase aguda da moléstia de Chagas. Rev Goiana Med 1980; 26:95-114.

5. Koeberle F. Patogenia da moléstia de Chagas. In: Cançado JR, editor. Doença de Chagas. Belo Horizonte (MG): Imprensa Oficial; 1968. p. 238-260.

6. Rezende JM. Manifestações digestivas da doença de Chagas. In: Dani R, Castro LP, editors. Gastroenterologia Clínica. 3a ed. Rio de Janeiro (RJ): Guanabara Koogan; 1993. p.1729-1755.

7. Adad SJ. Contribuição ao estudo da anatomia patológica e da patogênese do megacólon chagásico. [Tese]. [Uberaba (MG)]: Faculdade de Medicina do Triângulo Mineiro; 1996.

8. Rezende JM, Rassi A. Manifestações digestivas na fase aguda da doença de Chagas. In: Raia AA, editor. Manifestações digestivas da moléstia de Chagas. São Paulo (SP): Sarvier; 1983. p. 97-107.

9. Chagas C. Tripanosomiase americana. Forma aguda da molestia. Mem Inst Oswaldo Cruz 1916; 8:37-69.

10. Versiani O, Junqueira M. Adenopatias chagásicas. Brasil Med 1943; 57: 147-152.

11. Koeberle F. Enteropatias e enteromegalias. In: Simpósio Internacional sobre Enfermedad de Chagas. Sociedad Argentina Parasitologia. Buenos Aires 1972; p.77-84.

12. Becker PFL. Moléstia de Chagas aguda acidental (Por transfusão de sangue de doador chagásico). Rev Inst Med trop São Paulo 1975; 17:187-198. 
13. Dias JCP. Doença de Chagas em Bambuí, Minas Gerais, Brasil. Estudo clínico epidemiológico a partir da fase aguda, entre 1940 e 1982. [Tese]. [Belo Horizonte (MG)]: Faculdade de Medicina da Universidade Federal de Minas Gerais; 1982.

14. Lauar KM, Oliveira AR, Rezende JM. Valor do tempo de esvaziamento esofagiano no diagnóstico da esofagopatia chagásica (prova de retenção). Rev Goiana Med 1959; 5:97-102.

15. Rassi A, Rassi Jr A, Rassi GG. Fase aguda. In: Brenner Z, Andrade ZA, BarralNetto M, editors. Trypanosoma cruzi e Doença de Chagas. $2^{\mathrm{a}}$ ed. Rio de Janeiro (RJ): Guanabara Koogan. 2000; p. 231-245.

16. Rezende JM, Lauar KM, Oliveira AR. Aspectos clínicos e radiológicos da aperistalsis do esôfago. Rev Bras Gastroenterol 1960; 12:247-262.

17. Rezende JM. Forma digestiva da moléstia de Chagas. Rev Goiana Med 1959; 5:193-227.

18. Rassi A, Rezende JM, Doles J. Caso de doença de Chagas observado desde o período inicial da infecção, com aparecimento precoce de megaesôfago e megacólon. Rev Soc Bras Med Trop 1968; 2:303-315.

19. Lisboa AC. Sobre a forma congênita da doença de Chagas. Estudo anátomopatológico de 6 casos. Rev Inst Med Trop Sao Paulo 1960; 2:319-334.

20. Atias A, Almonte C. Megaesófago en un lactante con Enfermedad de Chagas probablemente congénita. Bol Chil Parasitol 1962; 17:46-48.

21. Tafuri WL, Lopes ER, Nunan B. Doença de Chagas congênita. Estudo clínicopatológico de um caso com sobrevida de seis meses. Rev Inst Med Trop Sao Paulo $1973 ; 15: 322-330$

22. Bitencourt ACL. Doença de Chagas congênita na Bahia. Rev Baiana Saude Publ 1984; 11:165-208.

23. Pompeu FR. Estudo longitudinal da doença de Chagas em trabalhadores rurais do município de Luz, MG (1976-1985). [Tese]. [Belo Horizonte (MG)]: Universidade Federal de Minas Gerais; 1990. (Citado por Castro et al ${ }^{24}$ ).

24. Castro C, Macedo V, Rezende JM, Prata A. Estudo radiológico longitudinal do esôfago, em área endêmica de doença de Chagas, em um período de seis anos. Rev Soc Bras Med Trop 1992; 25:225-230.

25. Hanani M, Ledder O, Yutkin V, Abu-Dalu R, Huang T-Y, Härtig W, et al Regeneration of myenteric plexus in the mouse colon after experimental denervation with benzalkonium chloride J Comparat Neurol 2003; 462 : 315-327. 